\title{
Synthesis of $\beta$-Nitroethanols via an Asymmetric Copper-Catalyzed Henry Reaction
}
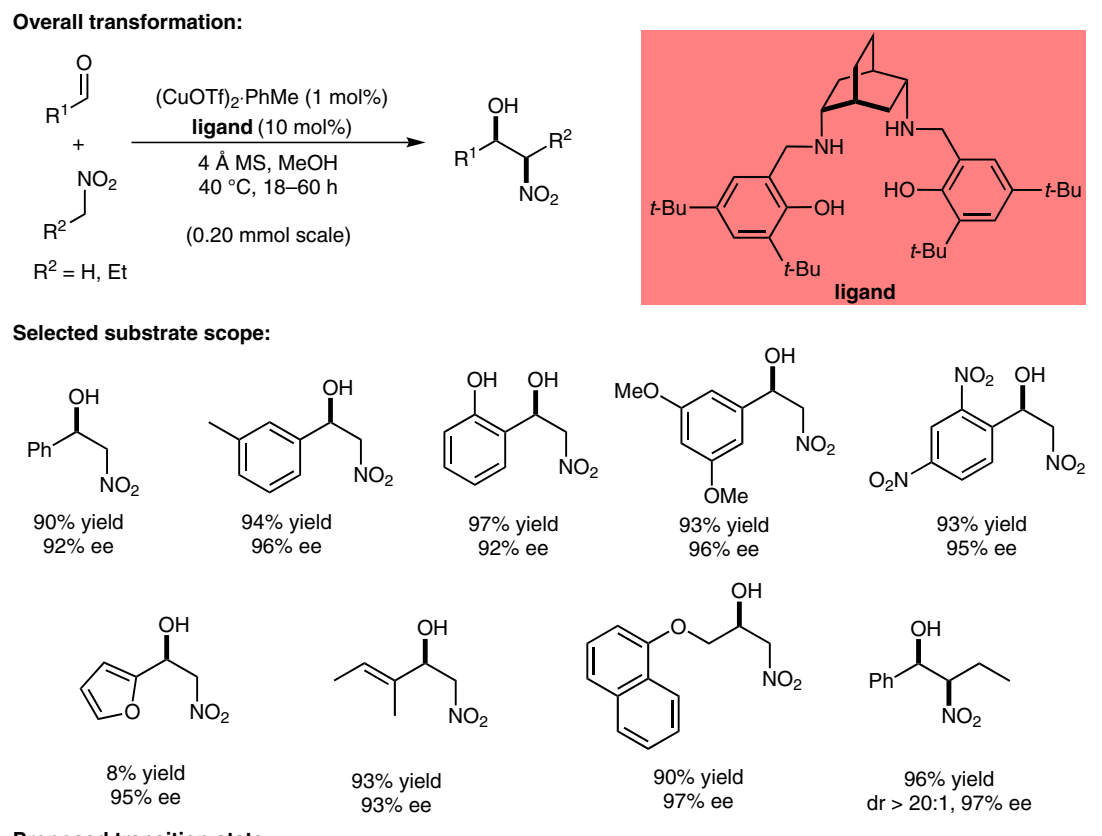

Proposed transition state:

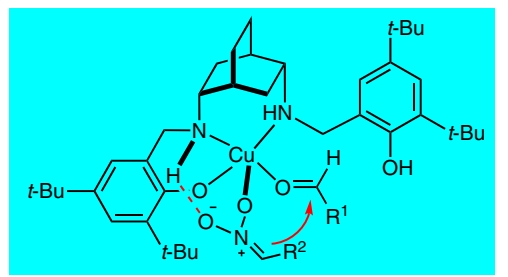

Significance: The nitro-aldol (or Henry) reaction is a valuable synthetic transformation which allows access to 1,2-amino alcohol precursors. The asymmetric version of this transformation has received considerable attention over the years, yet further development is needed to encompass a larger substrate scope. The authors report the use of a salen ligand, based on a chiral cis-2,5-diaminobicylo[2.2.2.]octane scaffold, to effect this transformation.
Comment: The authors report a copper-catalyzed asymmetric Henry reaction which provides $\beta$-nitro alcohols in excellent yields and enantioselectivities. Interestingly, when the authors employed the imine precursor of the ligand, the desired product was obtained in moderate yield and with low ee. To enhance the scope of this reaction the authors employed 1-nitropropane as the nitro enolate precursor and obtained the syn- $\beta$-nitro alcohols in high yield with excellent diastereo- and enantioselectivities. Furthermore, the authors highlighted this methodology in the synthesis of toliprolol, moprolol and propanolol, which are blocking agents of the $\beta$-adrenergic receptors.

SYNFACTS Contributors: Mark Lautens, David A. Petrone Synfacts 2013, 9(3), 0281 Published online: 15.02.2013 DOI: 10.1055/s-0032-1318262; Reg-No.: L00913SF 